Only $5 \%$ of epileptic patients in general are sensitive to photic stimulation. The video game is a specific provocative factor in predisposed "photosensitive" epileptic patients. These patients are also sensitive to television (40\%), flickering sunlight (35\%), disco lights (25\%), and striped patterns such as venetian blinds and escalators (10\%). In video game epilepsy, precipitating factors might include color, flashing lights or patterns, the cognitive content, and lack of sleep. Studies in the Netherlands show that viewing a "flashing" program on a $50 \mathrm{~Hz}$ television in close proximity to the set is the most provocative stimulus. A video game is more provocative than a picture with less color and movement. Children should be kept at a distance of at least 2 m from a television screen. (see Ped Neur Briefs Sept 1994).

\title{
EPILEPSY AND HYPERKINETIC BEHAVIOR
}

A 4-year-old boy with benign partial epilepsy and hyperkinetic behavior between seizures is reported from Sapporo Medical University, Japan. Hyperactivity was noted at age 3, and seizures began at 4 years 6 months. Attacks consisted of a terrified expression, crouching, and rubbing his forehead on the floor. They occurred in sleep and awake. An ictal EEG in sleep showed theta rhythm, predominant over the left hemisphere, followed by voltage depression, but no spike and wave complexes. Both seizures and hyperkinetic behavior responded to carbamazepine. The epilepsy was characterized as benign partial epilepsy with affective symptoms. (Wakai $S$ et al. Benign partial epilepsy with affective symptoms: Hyperkinetic behavior during interictal periods. Epilepsia July/Aug 1994;35:810-812). (Reprints: Dr S Wakai, Dept Pediatrics, Sapporo Medical University, School of Medicine, South 1 West 16, Chuo-ku, Sapporo, 060, Japan).

COMMENT. Behavioral and emotional disorder as a form of epilepsy is a controversial topic, and the response to antiepileptic drugs in treatment is not proof of epilepsy. This patient tends to support the concept of a specific epileptic syndrome, BPEAS, but the EEG evidence could be more convincing.

The following study provides evidence against the concept of a so-called "masked epilepsy" in some hyperkinetic children. A comparison of the emotional and behavioral problems of 53 children, aged 6-12 years, with epileptiform EEG discharges and those of children without this EEG abnormality showed no significant differences, in a study at the Tokyo Medical and Dental University; the Nihon University; Asai Hospital, Chiba; and National Center for Neurology and Psychiatry, Kodaira, Japan. The authors conclude that the emotional and behavioral problems are coincidental and not directly related to the epileptiform discharges. (Okubo Y et al. Epileptiform EEG discharges in healthy children: Prevalence, emotional and behavioral correlates, and genetic influences. Epilepsia July/Aug 1994;35:832-841). 